\title{
Are Patients with Potential Celiac Disease Really Potential? The Answer of Metabonomics
}

\author{
Patrizia Bernini, ${ }^{\dagger, \$}$ Ivano Bertini, ${ }^{,+,+\ddagger}$ Antonio Calabrò, ${ }^{\|, \perp}$ Giancarlo la Marca, ${ }^{\#, \nabla}$ Gabriele Lami," \\ Claudio Luchinat, ${ }^{*,+, \ddagger}$ Daniela Renzi, ${ }^{\prime \prime, \perp}$ and Leonardo Tenori ${ }^{\circledR}$ \\ Magnetic Resonance Center (CERM), University of Florence, Via L.Sacconi 6, 50019 Sesto Fiorentino, Italy, \\ Department of Chemistry, University of Florence, Via della Lastruccia 3, 50019 Sesto Fiorentino, Italy, FiorGen \\ Foundation, Via L. Sacconi 6, 50019 Sesto Fiorentino, Italy, Gastroenterology Unit, Department of Clinical \\ Pathophysiology, University of Florence, Viale Pieraccini 6, 50139 Florence, Italy, Tuscany Referral Center for \\ Adult Celiac Disease, Viale Morgagni 85, 50134 Florence, Italy, Department of Pharmacology, University of \\ Florence, Viale Pieraccini 6, 50139 Florence, Italy, and Mass Spectrometry, Clinical Chemistry and \\ Pharmacology Labs, Clinic of Pediatric Neurology, A. Meyer University Children's Hospital, Viale Pieraccini 24, \\ 50134 Florence, Italy
}

Received September 1, 2010

\begin{abstract}
Celiac disease (CD) is an autoimmune disorder caused by a permanent sensitivity to gluten in genetically susceptible individuals. Accurate diagnosis of $C D$ at an early stage and its treatment with a gluten-free diet (GFD) are important for optimum treatment and prognosis. Recently, by employing a noninvasive metabonomic approach, we have shown that CD has a well-defined metabonomic signature. Here we address potential CD patients, defined as subjects who do not have, and have never had, a jejunal biopsy consistent with clear $C D$, and yet have immunological abnormalities similar to those found in celiac patients. Sixty-one overt CD patients at diagnosis, 29 patients with potential CD, and 51 control subjects were examined by ${ }^{1} \mathrm{H}$ NMR of their serum and urine: out of 29 potential CD patients, 24 were classified as CD and 5 as control subjects. Potential CD largely shares the metabonomic signature of overt $C D$. Most metabolites found to be significantly different between control and CD subjects were also altered in potential $C D$. Our results demonstrate that metabolic alterations may precede the development of small intestinal villous atrophy and provide a further rationale for early institution of GFD in patients with potential $C D$, as recently suggested by prospective clinical studies.
\end{abstract}

Keywords: Potential Celiac Disease • Metabolomics • Metabonomics • Nuclear Magnetic Resonance

\section{Introduction}

Celiac disease (CD) is a common autoimmune disorder triggered by the ingestion of gluten and related prolamins, plant storage proteins found in wheat, barley, and rye, in genetically susceptible individuals. ${ }^{1}$ Patients display various degree of intestinal inflammation, ranging from mere increase in the number of intraepithelial lymphocytes (lymphocytic enteritis) to elongation of crypts (crypt hyperplasia), severe mononuclear cell infiltration of the lamina propria and overt villous atrophy. Accordingly, clinical manifestations range from completely

\footnotetext{
* Corresponding authors: Ivano Bertini, Magnetic Resonance Center (CERM), University of Florence, Via L. Sacconi 6, 50019 Sesto Fiorentino, Italy. E-mail, bertini@cerm.unifi.it; tel., 39-055-4574270; fax, 39-055-4574271. Claudio Luchinat, Magnetic Resonance Center (CERM), University of Florence, Via L. Sacconi 6, 50019 Sesto Fiorentino, Italy. E-mail, luchinat@ cerm.unifi.it; tel., 39-055-4574270; fax, 39-055-4574271.

${ }^{\dagger}$ Magnetic Resonance Center (CERM), University of Florence.

$\S$ FiorGen Foundation.

${ }^{\ddagger}$ Department of Chemistry, University of Florence.

" Department of Clinical Pathophysiology, University of Florence.

${ }^{\perp}$ Tuscany Referral Center for Adult Celiac Disease.

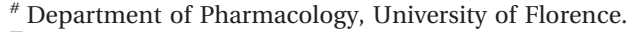

${ }^{\nabla}$ A. Meyer University Children's Hospital.
}

asymptomatic forms (so-called silent CD patients) to symptoms and signs of global malabsorption.

In the majority of cases, a gluten-free diet (GFD) leads to disappearance of clinical symptoms, recovery of normal mucosal histology, disappearance of the serological signs, and the prevention of complications, while reintroduction of gluten into the diet, at any stage, causes reactivation of the disease..$^{2-7}$

Small-bowel mucosal villous atrophy has traditionally been considered the conditio sine qua non for diagnosing CD. ${ }^{8}$ As a logical consequence, although there is a general consensus on dietary treatment for symptomatic subjects with enteropathy, it is not so for potential cases (i.e., patients with positive CDassociated serology, but no enteropathy). ${ }^{9}$ However, accumulating evidence suggests that celiac patients may suffer from gluten-sensitive symptoms even before villous atrophy has developed, ${ }^{10-13}$ and an increased risk of osteoporosis has been reported also for this group of patients. ${ }^{10}$

In a recent work, Bertini et al. have examined a cohort of CD patients, before and after gluten free diet GDF, and healthy controls, by ${ }^{1} \mathrm{H}$ NMR metabolic profiling of their serum and urine samples. ${ }^{14}$ Through multivariate analysis, they were able to demonstrate the existence of a clear metabonomic ${ }^{15}$ signa- 
ture of $\mathrm{CD}$ and to identify a number of serum and urine metabolites whose levels were significantly different in CD at diagnosis as compared to controls. The metabonomic signature as well the levels of most metabolites reverted to normal within 12 months of a strict GFD. The metabonomic fingerprint of $\mathrm{CD}$ has two components not directly linked to malabsorption: one related to energy metabolism, and the other related to alterations of gut microflora. Using this metabolic fingerprint, it is possible to make predictions about the CD status wit reasonably good accuracy (about $84 \%$ ).

These results, although extremely encouraging, are not particularly helpful from a merely diagnostic point of view, since serology by blood test already achieves a sensitivity of about $98 \%$ and a specificity of over $95 \% .{ }^{16}$ Moreover, professional guidelines recommend that a positive blood test should still be followed by an endoscopy, and this would pick up false positives as well as offer alternative explanations for the symptoms. ${ }^{17-19}$ However, the clear discrimination between patients and healthy subjects and patients and follow-up cases, achieved by metabolic fingerprinting, undoubtedly demonstrates the existence of a metabolic signature for CD both in serum and urine.

Under the inclusive definition of celiac disease, or glutensensitive enteropathy, the concepts of silent, latent, and potential $\mathrm{CD}$ have been introduced over 15 years ago by Professor Anne Ferguson. ${ }^{20}$ While silent CD is marked by severe damage to the jejunal mucosa in the absence of clinical symptoms, both latent and potential CD are characterized by jejunal mucosa that would be reported as normal by most clinical pathologists in an individual on a gluten-containing diet. Latent subjects sometimes in their life have had a flat jejunal biopsy which recovered on a gluten-free diet. Latent CD patients are often symptomatic; neither high titers of gliadin antibodies nor mucosal changes (including raised intraepithelial lymphocyte counts) are obligate features of latent CD, although the presence of elevated anti-endomysial antibodies (EmA) is probably the best predictor of progression toward villous atrophy. ${ }^{21}$

The term potential CD has been proposed for those subjects who, as opposed to latent CD patients, do not have, and have never had, a jejunal biopsy consistent with clear CD, and yet have immunological abnormalities similar to those found in celiac patients. Good markers of potential CD include the presence of serum endomysial antibodies, a high count of intraepithelial lymphocytes, and subtle pathological alteration such as increased density of intraepithelial lymphocytes expressing gamma delta $\mathrm{T}$ cell receptor, signs of activated mucosal cell-mediated immunity, celiac-like intestinal antibody pattern, and positive rectal gluten challenge. ${ }^{21}$

Our interest in potential CD subjects stems from the fact that they have all the typical immunoclinical features of clear $\mathrm{CD}$, in the absence of any apparent intestinal damage. The aim of this work is to investigate whether (and to what extent) potential CD patients share the same metabolic fingerprint of overt CD patients, thereby addressing an as yet open question: are the metabolic alterations associated to CD due to malabsorption (i.e., intestinal damage) or are they independent of mucosal injury and therefore intrinsic to the pathology? In other words, our aim is to clarify if we are effectively looking at a metabolic signature of CD or if we are simply following the metabolic modification due to intestinal malabsorption. To this end, a suitable cohort of potential CD subjects is ideal because, by definition, they do not have villous atrophy.
Recent prospective studies on both adults and children classified as potential CD patients have addressed the question of whether GFD prevents development of villous atrophy and/ or alleviates the symptoms. There is increasing evidence that GFD has a beneficial effect, although the effect can be more ${ }^{22,23}$ or less ${ }^{24}$ marked. The comparison of the fingerprint of potential CD subjects with overt CD subjects and healthy controls can thus contribute to the open debate about whether or not it is necessary to put these patients on a GFD.

\section{Materials and Methods}

Volunteers Recruitment and Samples Collection. The volunteers participating in this study were recruited by the Tuscany Referral Center for Adult CD. In total, 141 adult (35 males, 106 females, mean age $38.3 \pm 13.9$ years) participated in this study. Among the celiac group, 61 patients (10 males, 51 females, mean age $39.8 \pm 13.9$ years) were affected by overt $\mathrm{CD}$, and 29 were diagnosed as having potential CD (6 males, 23 females, mean age $34.4 \pm 14.3$ years). The diagnosis of CD was based on positive serology and confirmed by histological examination of small bowel biopsies taken from the distal part of the duodenum. Small intestinal mucosal damage was graduated according to the classification of Marsh modified by Oberhuber. ${ }^{25}$ According to the hystology, 7 patients with overt CD had partial villous atrophy (Marsh 3A), 7 subtotal villous atrophy (Marsh 3B), and 16 total villous atrophy (Marsh 3C). Patients with potential CD were all positive for anti-tissue transglutaminase antibodies (tTGA); all but one were also EmA positive; histological examination revealed an apparently normal mucosa (Marsh 0) in 9 subjects, just an increase in intraepithelial lymphocytes (Marsh 1) in 12 and increased intraepithelial lymphocytes coupled with crypt hyperplasia (Marsh 2) in 8 patients. The control group consisted of 51 nonceliac subjects (19 males, 32 females, mean age $36.6 \pm 14.2$ years): 34 (13 males, 21 females, mean age $37.6 \pm 14.8$ years) were healthy controls from the medical staff of the Gastrointestinal Unit and 17 (6 males, 11 females, mean age $30.9 \pm 12.5$ years) were patients with clinical suspicion of celiac disease; they were all EmA and tTGA negative. Therefore, for ethical reasons, the 34 controls that were from the medical staff did not undergo intestinal biopsy; in the remaining 17 cases, gastrointestinal endoscopy was performed only when it was considered necessary to the diagnosis. The clinical characteristics of all the subjects, including those examined in the previous work ${ }^{14}$ are reported in Supporting Table S1.

Each subject had fasted overnight, and urine and blood were collected in the morning preprandial. Venous blood samples were collected into plastic serum tubes $(6 \mathrm{~mL})$, with increased silica act clot activator, silicone-coated interior (Becton Dickinson, Plymouth, U.K.). Samples were allowed to clot by standing tubes vertically at room temperature $\left(22^{\circ} \mathrm{C}\right)$ for 60 min. Tubes were centrifuged at $1800 \mathrm{RCF}$ for $10 \mathrm{~min}$ at room temperature. Within 15 min of centrifugation, the supernatant (serum) was transferred in $500 \mu \mathrm{L}$ aliquots to prelabeled $1 \mathrm{~mL}$ cryovials (Bruker BioSpin, Milan, Italy). Three aliquots per patient were immediately frozen and stored at $-80{ }^{\circ} \mathrm{C}$ until used.

First morning preprandial urine void was used for the collection period. Patients were supplied with appropriate collection instructions and information on fasting, diet and medication restrictions when necessary. The urine samples were collected into prelabeled sterile collections cups. One milliliter of urine samples was transferred into prelabeled $1 \mathrm{~mL}$ 
sterile cryovials (Nalgene, Rochester, NY). Three aliquots per patient were immediately frozen and stored at $-80{ }^{\circ} \mathrm{C}$ until used.

Additionally, CD patients and healthy controls were asked to record their dietary intake and the use of any medication (either prescribed or self-administered) on the day before each visit, and to fast from midnight until blood samples and urine were collected the following morning.

Genotyping. Potential CD patients were analyzed for CDrelated genes following the procedure already reported. ${ }^{14}$ They were all genetically susceptible: in particular 22 were DQ2, 3 DQ8, and 4 DQ2.2 positive (i.e., they carried the HLA genes DRB1 *07, DQB1 *0202 and DQA1 *0201, see Supporting Table S2). In the course of our previous work, ${ }^{14}$ we had checked that among healthy subjects those who were DQ2/DQ8 positive were not statistically distinguishable from the negative ones, while they were distinguishable from the CD patients.

Antibody Testing. Anti-tissue transglutaminase antibodies were measured by a commercially available enzyme-linked immunosorbent assay kit (EutTG, Eurospital, Trieste, Italy) that employs human recombinant tTG as antigen; EmA were determined by indirect immunofluorescence, using tissue section of monkey esophagus (Eurospital), as previously described. $^{26}$

Citrulline Assay. Labeled standards of citrulline were purchased from Cambridge Isotope Laboratories, Andover, MA; a stock solution was made in methanol. The standard concentration was $500 \mu \mathrm{mol} / \mathrm{L}$. To obtain working solutions, daily dilutions (1:100) were made using methanol-water 85:15. All chemicals and solvents were of the highest purity available from commercial sources and were used without any further purification.

A dried serum spot was punched into a $1.5 \mathrm{~mL}$ tube or a 96 well plate and $200 \mu \mathrm{L}$ of methanol containing labeled standard was added. The sample was shaken on a vortex system for 20 min, and was then dried under a nitrogen flow at $50{ }^{\circ} \mathrm{C}$. The extracted amino acid was derivatized to butyl-ester using $n$-butanol and $\mathrm{HCl}(3 \mathrm{M})$ at $65{ }^{\circ} \mathrm{C}$ for $25 \mathrm{~min}$. After derivatization, the sample was dried under nitrogen flow at $55{ }^{\circ} \mathrm{C}$ and then recovered using $200 \mu \mathrm{L}$ of water/acetonitrile (1:1) containing $0.1 \%$ formic acid. A total of $40 \mu \mathrm{L}$ of the diluted sample was injected in FIA (Flow Injection Analysis) mode for the MS/MS experiments.

An Applied Biosystems-Sciex (Toronto, Canada) API 4000 triple-quadrupole mass spectrometer equipped with a TurboIonSpray source was employed for this study. The TurbolonSpray source was operated in positive ion mode with a needle potential of $+5900 \mathrm{~V}$ and with a "turbo" gas flow of $10 \mathrm{~L} / \mathrm{min}$ of air heated at $400{ }^{\circ} \mathrm{C}$ (nominal heating-gun temperature). Mass calibration and resolution adjustments on the resolving quadrupoles were performed automatically by using a PPG $10^{-7}$ $\mathrm{mol} / \mathrm{L}$ solution introduced via the built-in infusion pump. The peak width was set on both resolving quadrupoles at $0.7 \mathrm{Th}$ (measured at 1/2 height) for all MS and MS/MS experiments. Collision Activated Dissociation (CAD) MS/MS was performed in the LINAC Q2 collision cell, operating with 10 mTorr pressure of nitrogen as collision gas. The declustering potential (DP) and collision energy (CE) were automatically optimized for amino acids using the Analyst 1.3.2 software. The resulting DP was $+20 \mathrm{eV}$, and optimal CE was found to be $25 \mathrm{eV}$.

MS and MS/MS spectra were collected in continuous flow mode by connecting the infusion pump directly to the TurboIonSpray source. The quantitation experiments were per- formed using a Series 1100 Agilent Technologies (Waldbronn, Germany) CapPump coupled to an Agilent Micro ALS autosampler, both fully controlled from the API 4000 data system. Experimental flow rate was $50 \mu \mathrm{L} / \mathrm{min}$ using water/acetonitrile (1:1) mixture containing $0.1 \%$ formic acid. The eluent from the column was directed to the TurboIonSpray probe. The acquired data were processed using the Analyst 1.4.1 proprietary software including the "Explore" option (for chromatographic and spectral interpretation) and the ChemoView software (for quantitative information generation).

NMR Sample Preparation. Frozen serum samples were thawed at room temperature and shaken before use. A total of $300 \mu \mathrm{L}$ of a phosphate sodium buffer $\left(70 \mathrm{mM} \mathrm{Na}_{2} \mathrm{HPO}_{4} ; 20 \%\right.$ (v/v) ${ }^{2} \mathrm{H}_{2} \mathrm{O} ; 0.025 \%$ (w/v) $\mathrm{NaN}_{3} ; 0.8 \%$ (w/v) sodium trimethylsilyl $\left[2,2,3,3-{ }^{2} \mathrm{H}_{4}\right]$ propionate (TSP); $\mathrm{pH} 7.4$ ) was added to $300 \mu \mathrm{L}$ of each serum sample, and the mixture was homogenized by vortexing for $30 \mathrm{~s}$. A total of $550 \mu \mathrm{L}$ of this mixture was transferred into a $4.25 \mathrm{~mm}$ NMR tube (Bruker BioSpin srl) for analysis.

Frozen urine samples were thawed at room temperature and shaken before use. Samples were centrifuged at $1.4 \times 10^{4} \mathrm{~g}$ for $5 \mathrm{~min}$ and $540 \mu \mathrm{L}$ of the supernatant was added to $60 \mu \mathrm{L}$ of sodium phosphate buffer $\left(0.2 \mathrm{M} \mathrm{Na}_{2} \mathrm{HPO}_{4} ; 0.2 \mathrm{M} \mathrm{NaH}_{2} \mathrm{PO}_{4}\right.$ in $100 \%{ }^{2} \mathrm{H}_{2} \mathrm{O}$; pH 7.0) also containing $10 \mathrm{mM}$ TSP, and $30 \mathrm{mM}$ $\mathrm{NaN}_{3}$. A total of $450 \mu \mathrm{L}$ of the mixture was pipetted into 4.25 mm NMR tubes (Bruker BioSpin srl).

NMR Analysis. ${ }^{1} \mathrm{H}$ NMR spectra for all samples were acquired using a Bruker $600 \mathrm{MHz}$ spectrometer (Bruker BioSpin) operating at $600.13 \mathrm{MHz}$ proton Larmor frequency and equipped with a $5 \mathrm{~mm}$ CPTCI ${ }^{1} \mathrm{H}-{ }^{13} \mathrm{C} /{ }^{31} \mathrm{P}-{ }^{2} \mathrm{H}$ cryo-probe including a $z$-axis gradient coil, an automatic tuning-matching (ATM) and an automatic sample changer. A PT 100 thermocouple provided temperature stabilization at the level of approximately $0.1 \mathrm{~K}$ for the sample. Before measurement, samples were kept for at least $3 \mathrm{~min}$ inside the NMR probehead, for temperature equilibration $(300 \mathrm{~K})$.

For each urine sample, a one-dimensional NMR spectrum was acquired with water peak suppression using a standard pulse sequence (NOESYpresat; Bruker), using 64 free induction decays (FIDs), 64k data points, a spectral width of $12019 \mathrm{~Hz}$, an acquisition time of $2.7 \mathrm{~s}$, a relaxation delay of $4 \mathrm{~s}$, and a mixing time of $100 \mathrm{~ms}$.

One-dimensional spectra of serum samples were acquired using a Carr-Purcell-Meiboom-Gill (CPMG; Bruker) spin-echo sequence to suppress signals arising from high molecular weight molecules and a standard pulse sequence (NOESYpresat, same acquisition parameters as in the case of urine samples).

Spectral Processing. Free induction decays were multiplied by an exponential function equivalent to a $1.0 \mathrm{~Hz}$ linebroadening factor before applying Fourier transform. Transformed spectra were manually corrected for phase and baseline distortions and calibrated (TMSP peak at $0.00 \mathrm{ppm}$ ) using TopSpin (Version 2.1, Bruker). Each 1-D spectrum in the range between 0.02 and $10.00 \mathrm{ppm}$ was segmented into $0.02-\mathrm{ppm}$ chemical shift bins, and the corresponding spectral areas were integrated using AMIX software (Bruker BioSpin). Regions between 6.0 and $4.5 \mathrm{ppm}$ containing residual water and urea signals were removed. The total spectral area was calculated on the remaining bins and normalization was carried out on the data prior to pattern recognition.

Statistical Analysis. Data reduction was obtained by means of Kernel Orthogonal Projection to Latent Structure (KOPLS), ${ }^{27}$ 
using the algorithm implemented in the R-library "kopls"28 using a Gaussian kernel.

For the purpose of classification, we used the Support Vector Machines (SVM) method $^{29}$ applied on the KOPLS scores. The "libsvm" module (LIBSVM: a Library for Support Vector Machines) of the R-library "e1071" was used. ${ }^{30}$

The statistical procedure used is a combination of KOPLS for data reduction (i.e., to reduce the initial number of variables and to have a clear depiction of the class separation) followed by SVM on the KOPLS scores. SVM are used with the default "C-classification" settings.

Accuracy, specificity, and sensitivity were estimated using standard definitions. The global accuracy for classification was assessed by means of double cross-validation scheme. ${ }^{31}$ The original data set was spit in a training set $(90 \%)$ and a test set $(10 \%)$ prior to any other step of statistical analysis. Parameter selection was carried out by 3 -fold cross-validation on the $90 \%$ training set. The whole procedure was repeated 100 times

A
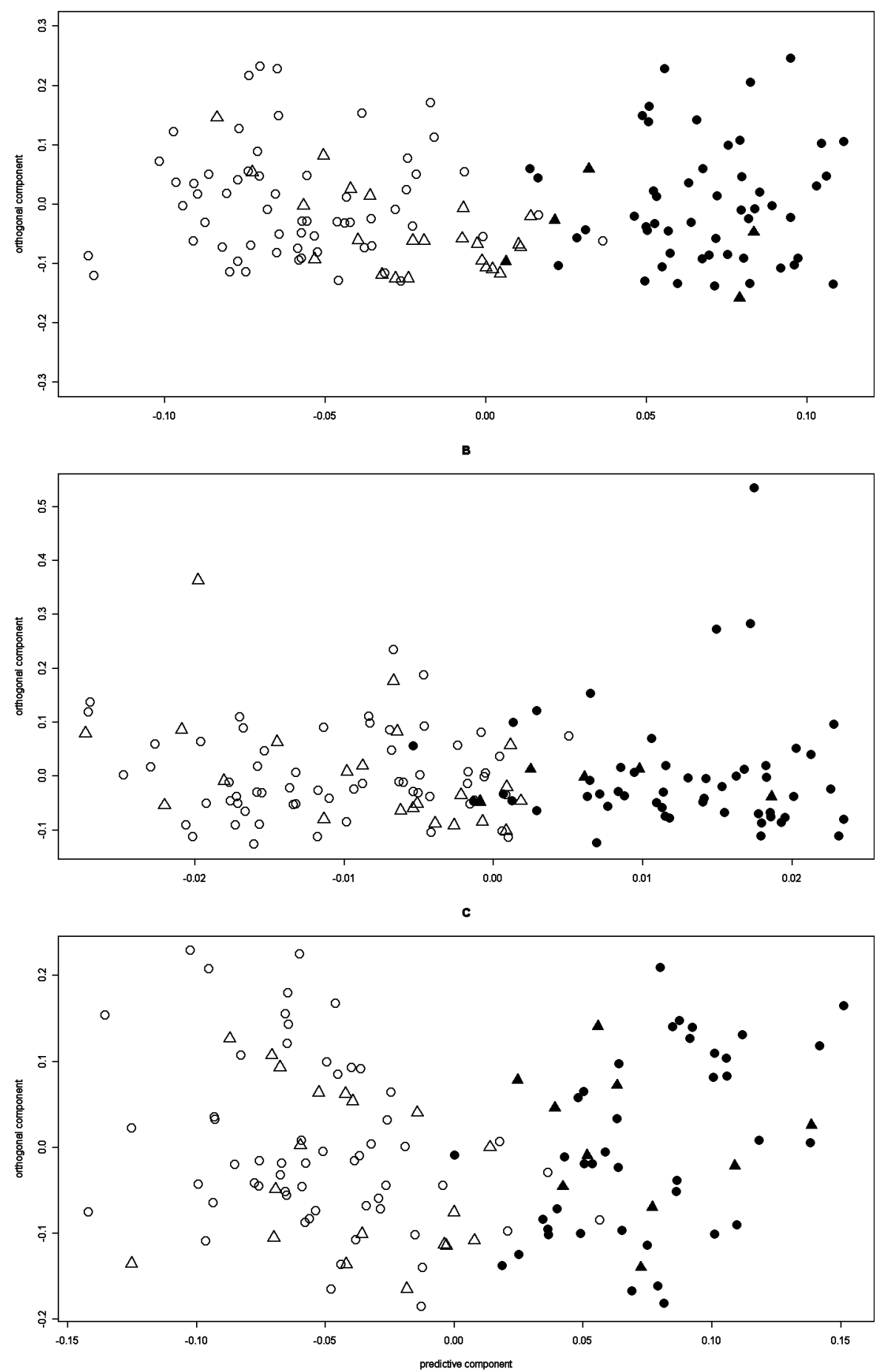

Figure 1. Clusterization of overt CD patients (open circles) and healthy controls (filled circles) obtained with kopls for cpmg serum spectra (A), NOESY serum spectra (B), and NOESY urine spectra (C). Potential CD patients (triangles) are projected on the kopls component, predicted by SVM, and filled or not accordingly to the results. 
Table 1. Cross-Validation Classification Results Obtained Using CPMG and NOESY Serum Spectra and NOESY Urine Spectra of Overt CD Patients and Nonceliac Controls (NC)

\begin{tabular}{|c|c|c|c|}
\hline & overt CD & NC & accuracy \\
\hline \multicolumn{4}{|c|}{ Serum CPMG Spectra } \\
\hline overt CD & $80.5 \%$ & $19.5 \%$ & \multirow{2}{*}{$81.2 \%$} \\
\hline $\mathrm{NC}$ & $18.0 \%$ & $82.0 \%$ & \\
\hline \multicolumn{4}{|c|}{ Serum NOESY Spectra } \\
\hline overt CD & $77.4 \%$ & $22.6 \%$ & \multirow{2}{*}{$75.5 \%$} \\
\hline $\mathrm{NC}$ & $26.7 \%$ & $73.3 \%$ & \\
\hline \multicolumn{4}{|c|}{ Urine NOESY Spectra } \\
\hline overt CD & $77.5 \%$ & $22.5 \%$ & \multirow{2}{*}{$74.7 \%$} \\
\hline $\mathrm{NC}$ & $29.0 \%$ & $71.0 \%$ & \\
\hline
\end{tabular}

inside a Monte Carlo cross-validation scheme. From this procedure, we obtain unbiased generalization error estimation. To avoid overtraining and to speed up the calculations, in the inner loop of the cross-validation (parameter selection), the SVM was kept with the default settings, varying only the number of components and the kernel sigma parameter for KOPLS.

To assess which buckets (that is, resonance peaks) were significantly different between different groups, a univariate wilcoxon test was used. A $P$-value $\leq 0.05$ was considered statistically significant. The buckets whose values appeared to be significantly different from the above statistical analysis were manually checked to identify the associated resonance peak(s). All resonances of interest were then manually checked and signals were assigned on template one-dimensional NMR profiles by using matching routines of AMIX 6.8 (Bruker BioSpin) in combination with the BBIOREFCODE (Bruker BioSpin) reference database and published literature when available. All calculations were made using homemade scripts written in R language. ${ }^{32}$ Serum citrulline concentrations in the three groups were analyzed by two-way ANOVA, followed by Tukey's multiple comparison test, and a $P$-value of less than 0.05 was accepted to denote statistical significance.

Ethical Issue. The study was approved by the Internal Review Board of the Department of Clinical Pathophysiology of the University of Florence, and informed consent was obtained from all patients and healthy volunteers.

\section{Results}

The clusterization of controls and overt untreated CD patients for the three sets of experiments (serum CPMG, serum NOESY, and urine NOESY) is shown in Figure 1. It appears that the best clusterization is provided by the serum CPMG spectra.

The statistical models applied proved to be effective in discriminating between patients and control subjects. These results are in line with our previous work, ${ }^{14}$ where for the first time the existence of a metabolic fingerprint of celiac disease was demonstrated, both in serum and urine. Although the best recognition accuracy is slightly less than previously reported (81.2 vs 83.4 for serum CPMG spectra), these new data undoubtedly confirm the existence of the metabolic signature of CD, both in serum and in urine. In Table 1, the values for classification accuracy for each experiment are reported.

If the potential CD patients are treated as blind samples and are subjected to classification by the previous statistical model, 24 out of 29 are classified as CD patients using CPMG serum spectra. Those results strongly suggest that potential CD subjects are more similar to overt CD than to healthy controls.
Table 2. Cross-Validation Classification Results Obtained Using CPMG and NOESY Serum Spectra and NOESY Urine Spectra of CD Patients and Potential CD Subjects

\begin{tabular}{|c|c|c|c|}
\hline & overt CD & potential CD & accuracy \\
\hline \multicolumn{4}{|c|}{ Serum CPMG Spectra } \\
\hline overt CD & $71.1 \%$ & $28.9 \%$ & \multirow{2}{*}{$57.8 \%$} \\
\hline potential CD & $70.2 \%$ & $29.8 \%$ & \\
\hline \multicolumn{4}{|c|}{ Serum NOESY Spectra } \\
\hline overt CD & $67.6 \%$ & $32.3 \%$ & \multirow{2}{*}{$55.2 \%$} \\
\hline potential CD & $71.1 \%$ & $28.9 \%$ & \\
\hline \multicolumn{4}{|c|}{ Urine NOESY Spectra } \\
\hline overt CD & $81.4 \%$ & $18.6 \%$ & \multirow{2}{*}{$57.4 \%$} \\
\hline potential CD & $89.7 \%$ & $10.3 \%$ & \\
\hline
\end{tabular}

Moreover, when the statistical analysis is used to discriminate potential from overt CD patients, the accuracy is significantly lower (57.8\%). Furthermore, as shown in Table 2, this result is highly asymmetric with high sensitivity (>70\%) but poor specificity (ca. 20\%). Again potential CD subjects show a behavior that is much more similar to overt CD.

When comparing spectra of serum samples of overt untreated CD patients and nonceliac controls, we find that CD patients are characterized $(P<0.05)$ by lower levels of choline, creatinine, isoleucine, lactate, leucine, methionine, valine, lipids, and glycoproteins (from their $\mathrm{N}$-acetyl signal) and higher levels of glucose, citrate, serine, and phenylalanine.

In the case of urine, overt CD patients are characterized by higher levels of acetoacetate, choline, glutamate/glutamine, indoxyl sulfate (IS), meta-hydroxyphenyl-propioni acid $(\mathrm{mH}-$ PPA), phenylacetylglycine (PAG), 3-hydroxybutyric acid, methylamine, valeric acid, sarcosine, $p$-cresolsulfate, and 1-methylnicotinamide. These data are summarized in Table 3. Even though minor differences are present (due to the different data set and slightly different statistical analysis), this pattern is coherent with the previous work and corroborates the previous findings. $^{14}$

Comparing the serum spectra of overt CD with those of potential $\mathrm{CD}$, we find that potential CD patients are characterized by lower levels of dimethylglycine and higher levels of glutamate and lipids $(P<0.05)$; no other metabolites are found significantly different in serum. In urine, more differences are found, in particular potential CD patients shows lower levels of glutamate/glutamine, IS, mHPPA, PAG, $p$-cresolsulfate, and citrate (see Table 3).

The metabolic differences between potential CD and nonceliac controls in serum are quite similar to those between overt $\mathrm{CD}$ and controls: potential CD subjects have lower levels of creatinine, leucine, dimethylglycine, arginine, and lipids and higher levels of glucose, proline, and glutamate. In urine, potential CD subjects show lower levels of hippurate and higher levels of dimethylamine, 1-methylnicotinamide, and citrate. These data are summarized in Table 3.

Several of the potential CD patients analyzed here were put on a GFD, although solely based on medical considerations and not on the results from metabolomics. At the present stage, we have been able to examine again 13 of them, after an average period of GFD of 6 months (Supporting Table S2): all but three had negative TGA. By processing the follow-up serum samples with the same statistical model built for the discrimination, we found that all of them, except for one of the three positive to TGA, had reverted to a normal metabonomic profile.

In addition to metabolomic analysis, with the aim of obtaining more information able to better characterize the clinical 
Table 3. Metabolites Found To Be Statistically Different $(P<0.05)$ in Sera $(A)$ and Urine $(B)$ of Untreated Overt CD Patients, Potential CD Patients, and Nonceliac Controls (CD) ${ }^{a}$

\begin{tabular}{cccc}
\hline & CD patients & potential & overt CD vs \\
metabolite & vs NC & CD vs NC & potential CD \\
\hline
\end{tabular}

\section{Choline}

Creatinine

Glucose

Glycoproteins

Isoleucine

Lactate

Leucine

Lipids

Methionine

Valine

Citrate

Serine

Phenilalanine

Glutamate

Dimethylglycine

Proline

Arginine

(A) Serum

Acetoacetate

Choline

Glutamate/Glutamine

Indoxyl Sulfate

mHPPA

PAG

3-OH-butirate

Methylamine

Valerate

Sarcosine

p-cresolsulfate

1-methylnicotinammide

Citrate

Hippurate

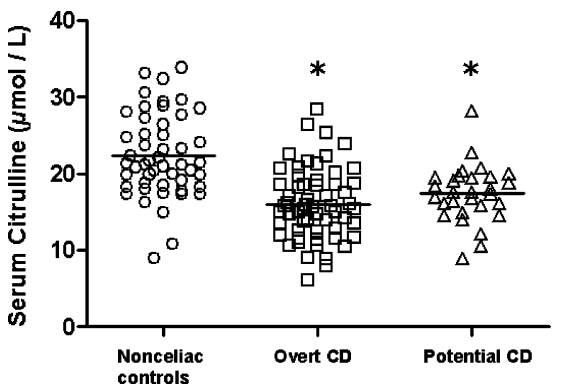

Figure 2. Serum citrulline concentrations in the three groups of subjects. In overt and potential $C D$, the mean serum citrulline concentration was significantly lower $(15.97+4.62$ SD and 17.38 $+3.70 \mathrm{SD})$ than in nonceliac controls $\left(22.30+5.39 \mathrm{SD},{ }^{*} p\right.$ $<0.001)$. One-way analysis of variance followed by Tukey's post test was applied to the results.

so we are not proposing an alternative diagnostic test for the screening of difficult cases, sometimes challenging also for expert clinicians. Rather, this exercise is useful to have a qualitative answer to the title question. Using CPMG serum spectra, the result of the prediction is remarkably clear: out of 29 potential CD subjects, 24 are classified as CD and 5 are classified as controls. Furthermore, three of the latter are located near the cluster boundary (e.g., they are borderline subjects) and only two are clearly classified as controls (Figure 1A). Although it is not possible to give a rationale for these differences, the answer of metabolomics is straightforward: potential CD subjects are extremely similar to overt CD patients, at least in their metabolic profile.

If we build a statistical model optimized for the discrimination between celiac patients and potential subjects, the classification accuracy is very low: $57.8 \%$ for serum CPMG spectra, $55.1 \%$ for serum NOESY spectra, and $57.4 \%$ for urine. In all cases, we have high sensitivity but a high rate of false discovery, so the specificity is very poor $(29 \%, 29 \%$, and $10 \%)$. In other words, it is impossible to separate overt CD from potential CD subjects, based on metabolomics.

The analysis of the metabolic profiles reveals interestingly similar behavior of overt CD and potential CD. Only few serum metabolites differentiate potential from overt $\mathrm{CD}$, and none of these metabolites are relative to energy metabolism. In particular, it appears that, as in untreated CD patients, glycolysis is somehow impaired. Impairment of glycolysis explains both a lowering of lactate levels and an increase of glucose levels in blood. This is confirmed by the metabolic pattern that differentiates potential $\mathrm{CD}$ and nonceliac controls: most of the significant metabolites are the same that differentiate overt CD from control group.

In urine, there are many more metabolites that differentiate potential CD from overt CD than from controls. Especially, the key differences are in the metabolites originating from the gut microflora (m-HPPA, IS, and PAG), suggesting a relationship between overt CD, villous atrophy, and bacterial consortia of the host. It is also reported ${ }^{38}$ that particular bacterial strains may work in conjunction with gluten to cause an increased immune response which is responsible of the development of CD. A distinctive 'microbial signature' in celiac pediatric patients is also reported. ${ }^{39}$

The similarity of the metabolic pattern in serum, and the dissimilarity in urine, between overt and potential CD subjects allow us to hypothesize that while the alterations in the urine profile may follow intestinal damage, gluten intolerance exists as such before an evident intestinal damage occurs. 
To clarify this aspect and to shed more light on the present findings, we performed a serum citrulline assay. In humans, citrulline is an amino acid involved in intermediary metabolism and is not incorporated in proteins. ${ }^{40-42}$ Circulating citrulline is mainly produced by enterocytes of the small bowel. ${ }^{43}$ For this reason, plasma or serum citrulline concentration has been proposed as a biomarker of residual small bowel mass and function. ${ }^{44}$

Our data, in line with previous findings, clearly show that citrulline levels in the CD patients are significantly lower than in the control group. But, more strikingly, also potential CD subjects, that from endoscopy do not show any manifest sign of villous atrophy, have lower levels of citrulline than controls. Indeed no statistically significant difference is found between citrulline levels in CD patients and potential CD subjects (Figure 2).

According to clinical experience, if a celiac subject has a positive antibody test but does not show macroscopic alterations of intestinal mucosa, there can be only two possibilities: (1) a (limited) intestinal damage is present but the endoscopy was not able to detect it (i.e., we admit some error in the sampling procedure) or (2) the intestinal mucosa is really intact and the subject is a so-called "potential CD subject".

The interpretation of our data (both from metabonomics and from citrulline assay) suggests a third option: potential CD subjects are, indeed, not potential at all. They already experience some subtle alteration of the enterocytes (at the microscopic functional level but not at the macroscopic level) and metabolically appear similar to overt CD also without any histological evidence of intestinal damage. If this hypothesis holds, the core result from our investigation would be that metabolomics can detect CD also when its clinical manifestation is not fully evident. Moreover, these findings may bear the important medical implication that the metabolic and enterocyte alterations of $\mathrm{CD}$ arise before macroscopic intestinal damage and/or villous atrophy and precede the observable modification of jejunal mucosa. In this context, the observation that potential CD subjects are characterized by a similar serum metabonomic fingerprint as overt $\mathrm{CD}$ patients provides a further, more biological, rationale for the medical evidencebased choice of putting potential CD subjects on a GFD. Moreover, if the metabolic changes are (at least partially) independent from the bowel malabsorption (i.e., metabolites of gut microflora and energetic pathway), a deeper analysis of this fingerprint can be helpful to infer more information on the biochemistry of the development of the disease.

Acknowledgment. This work was partly supported by a fellowship from Boehringer Ingelheim Italia (to L.T. through the FiorGen Foundation). The authors declare no competing financial interest.

Supporting Information Available: Tables of demographic and clinical data on study patients; demographic data, genotyping, antibodies levels and metabolomic classification of the 29 potential CD subjects. This material is available free of charge via the Internet at http://pubs.acs.org.

\section{References}

(1) Kagnoff, M. F. Celiac disease: pathogenesis of a model immunogenetic disease. J. Clin. Invest. 2007, 117, 41-49.

(2) Trier, J. S. Celiac sprue. N. Engl. J. Med. 1991, 325, 1709-1719.

(3) Schuppan, D. Current concepts of celiac disease pathogenesis. Gastroenterology 2000, 119, 234-242.
(4) Farrell, R. J.; Kelly, C. P. Celiac sprue. N. Engl. J. Med. 2002, 346, $180-188$.

(5) Green, P. H.; Jabri, B. Coeliac disease. Lancet 2003, 362, 383-391.

(6) Green, P. H.; Cellier, C. Celiac disease. N. Engl. J. Med. 2007, 357, 1731-1743.

(7) Di Sabatino, A.; Corazza, G. R. Coeliac disease. Lancet 2009, 373, $1480-1493$.

(8) Troncone, R.; Auricchio, R.; Granata, V. Issues related to glutenfree diet in coeliac disease. Curr. Opin. Clin. Nutr. Metab. Care 2008, 11, 329-333.

(9) Setty, M.; Hormaza, L.; Guandalini, S. Celiac disease: risk assessment, diagnosis, and monitoring. Mol. Diagn. Ther. 2008, 12, 289298.

(10) Kaukinen, K.; Mäki, M.; Partanen, J.; Sievänen, H.; Collin, P. Celiac disease without villous atrophy. Revision of criteria called for. Dig. Dis. Sci. 2001, 46, 879-887.

(11) Kaukinen, K.; Peräaho, M.; Collin, P.; Partanen, J.; Woolley, N.; Kaartinen, T.; Nuutinen, T.; Halttunen, T.; Mäki, M.; KorponaySzabo, I. Small bowel mucosal transglutaminase 2-specific IgA deposits in coeliac disease without villous atrophy: a prospective and randomized study. Scand. J. Gastroenterol. 2005, 40, 564-572.

(12) Troncone, R. Latent coeliac disease in Italy. Acta Paediatr. 1995, $84,1252-7$.

(13) Matysiak-Budnik, T.; Malamut, G.; de Serre, N. P.; Grosdidier, E.; Seguier, S.; Brousse, N.; Caillat-Zucman, S.; Cerf-Bensussan, N.; Schmitz, J.; Cellier, C. Long-term follow-up of 61 patients diagnosed in childhood: evolution towards latency is possible on a normal diet. Gut 2007, 56, 1379-86.

(14) Bertini, I.; Calabrò, A.; De Carli, V.; Luchinat, C.; Nepi, S.; Porfirio, B.; Renzi, D.; Saccenti, E.; Tenori, L. The metabonomic signature of celiac disease. J. Proteome Res. 2009, 8, 170-177.

(15) Nicholson, J. K.; Lindon, J. C.; Holmes, E. 'Metabonomics': understanding the metabolic responses of living systems to pathophysiological stimuli via multivariate statistical analysis of biological NMR spectroscopic data. Xenobiotica 1999, 29, 11811189.

(16) Fasano, A.; Catassi, C. Current approaches to diagnosis and treatment of celiac disease: an evolving spectrum. Gastroenterology 2001, 12, 1527-1528.

(17) Jackson Allen, P. L. Guidelines for the diagnosis and treatment of celiac disease in children. Pediatr. Nurs. 2004, 30, 473-476.

(18) Cellier, C.; Green, P. H.; Collin, P.; Murray, J. ICCE consensus for celiac disease. Endoscopy 2005, 37, 1055-1059.

(19) Hill, I. D.; Dirks, M. H.; Liptak, G. S.; Colletti, R. B.; Fasano, A.; Guandalini, S.; Hoffenberg, E. J.; Horvath, K.; Murray, J. A.; Pivor, M.; Seidman, E. G. North American Society for Pediatric Gastroenterology, Hepatology and Nutrition. Guideline for the diagnosis and treatment of celiac disease in children: recommendations of the North American Society for Pediatric Gastroenterology, Hepatology and Nutrition. J. Pediatr. Gastroenterol. Nutr. 2005, 40, 119.

(20) Ferguson, A.; Arranz, E.; O'Mahony, S. Clinical and pathological spectrum of coeliac disease: active, silent, latent and potential. Gut 1993, 34, 150-151.

(21) Troncone, R.; Greco, L.; Mayer, M.; Paparo, M.; Caputo, N.; Micillo, M.; Mugione, P.; Auricchio, S. Latent and potential coeliac disease. Acta Paediatr. Supp. 1996, l412, 10-14.

(22) Kurppa, K.; Ashorn, M.; Iltanen, S.; Koskinen, L. L.; Saavalainen, P.; Koskinen, O.; Mäki, M.; Kaukinen, K. Celiac disease without villous atrophy in children: a prospective study. J. Pediatr. 2010, 157 (3), 373-380.

(23) Kurppa, K.; Collin, P.; Viljamaa, M.; Haimila, K.; Saavalainen, P.; Partanen, J.; Laurila, K.; Huhtala, H.; Paasikivi, K.; Mäki, M.; Kaukinen, K. Diagnosing mild enteropathy celiac disease: a randomized, controlled clinical study. Gastroenterology 2009, 136 (3), 816-823.

(24) Tosco, A.; Salvati, V. M.; Auricchio, R.; Maglio, M.; Borrelli, M.; Corazzo, A.; Paparo, F.; Beffardi, M.; Esposito, A.; D’Adamo, G.; Malamisura, B.; Greco, L.; Troncone, R. Most children with potential celiac disease are healthy but 1 third of them develop villous atrophy at 3-years follow-up Clin. Gastroenterol. Hepatol. [Online early access]. DOI: 10.1016/j.cgh.2010.09.006. Published online: Sept 17, 2010.

(25) Oberhuber, G.; Granditsch, G.; Vogelsang, H. The histopathology of coeliac disease: time for a standardized report scheme for pathologists. Eur. J. Gastroenterol. Hepatol. 1999, 11, 1185-1194.

(26) Matà, S.; Renzi, D.; Pinto, F.; Calabrò, A. Anti-tissue transglutaminase IgA antibodies in peripheral neuropathy and motor neuronopathy. Acta Neurol. Scand. 2006, 114, 54-58. 
(27) Rantalainen, M.; Bylesjö, M.; Cloarec, O.; Nicholson, J. K.; Holmes, E.; Trygg, J. Kernel-based orthogonal projections to latent structures (K-OPLS). J. Chemom. 2007, 21, 376-385.

(28) Bylesjö, M.; Rantalainen, M.; Nicholson, J. K.; Holmes, E.; Trygg, J. K-OPLS package: kernel-based orthogonal projections to latent structures for prediction and interpretation in feature space. $B M C$ Bioinf. 2008, 9, 106-110.

(29) Vapnik, V. N. The Nature of Statistical Learning Theory; SpringerVerlag: New York, 1995.

(30) Chang C.-C.; Lin, C.-J.; LIBSVM: a Library for Support Vector Machines, 2001. Software available at http://www.csie.ntu.edu.tw/ $\sim$ cjlin/libsvm.

(31) Westerhuis, J. A.; Hoefsloot, H. C. J.; Smit, S.; Vis, D. J.; Smilze, A. K.; van Velzen, E. J. J.; van Duijnhoven, J. P. M.; van Dorsten, F. A. Assessment of PLSDA cross validation. Metabolomics 2008, $4,81-89$.

(32) Ihaka, R.; Gentleman, R. R: A language for data analysis and graphic. J. Comput. Stat. Graph. 1996, 5, 299-314.

(33) Crenn, P.; Coudray-Lucas, C.; Thuillier, F.; Cynober, L.; Messing, B. Postabsorptive plasma citrulline concentration is a marker of absorptive enterocyte mass and intestinal failure in humans. Gastroenterology 2000, 119, 1496-505.

(34) Crenn, P.; Vahedi, K.; Lavergne-Slove, A.; Cynober, L.; Matuchansky, C.; Messing, B. Plasma citrulline: a marker of enterocyte mass in villous atrophy-associated small bowel disease. Gastroenterology 2003, 124, 1210-1219.

(35) Blijlevens, N. M.; Lutgens, L. C.; Schattenberg, A. V.; Donnelly, J. P. Citrulline: a potentially simple quantitative marker of intestinal epithelial damage following myeloablative therapy. Bone Marrow Transplant. 2004, 34, 193-196.

(36) Pappas, P. A.; Tzakis, A. G.; Saudubray, J. M. Trends in serum citrulline and acute rejection among recipients of small bowel transplants. Transplant Proc. 2004, 36, 345-347.

(37) Miceli, E.; Poggi, N.; Missanelli, A.; Bianchi, P.; Moratti, R.; Corazza, G. R. Is serum citrulline measurement clinically useful in coeliac disease. Intern. Emerg. Med. 2008, 3, 233-236.

(38) Sollid, M. L.; Gray, G. M. A role for bacteria in celiac disease. Am. J. Gastroenterol. 2004, 99, 894-904.

(39) Schippa, S.; Iebba, V.; Barbato, M.; di Nardo, G.; Totino, V.; Proietti Checchi, M.; Longhi, C.; Maiella, G.; Cucchiara, S.; Conte, M. P. A distinctive 'microbial signature' in celiac pediatric patients. BMC Microbiol. 2010, 10, 175.

(40) Windmueller, H. G.; Spaeth, A. E. Source and fate of circulating citrulline. Am. J. Physiol. 1981, 241, 473-480.

(41) Wakabayashi, Y.; Jones, M. E. Pyrroline-5-carboxylate synthesis from glutamate by rat intestinal mucosa. J. Biol. Chem. 1983, 258, 3865-3872.

(42) Wu, G. Intestinal mucosal amino acid catabolism. J. Nutr. 1998, 128, 1249-1252.

(43) Wakabayashi, Y.; Yamada, E.; Hasegawa, T.; Yamada, R. Enzymological evidence for the indispensability of small intestine in the synthesis of arginine from glutamate.I. Pyrroline-5-carboxylate synthase. Arch. Biochem. Biophys. 1991, 291, 1-8.

(44) Crenn, P.; Messing, B.; Cynober, L. Citrulline as a biomarker of intestinal failure due to enterocyte mass reduction. Clin Nutr. 2008, 27, 328-339.

PR100896S 